15

\title{
Резонансная микроволновая спектроскопия полупроводников с микронным разрешением
}

\author{
(C) А.Н. Резник, Н.В. Востоков
}

Институт фозиики микроструктур РАН, 603950 Нижний Новгород, Россия

e-mail: reznik@ipm.sci-nnov.ru

Поступило в Редакцию 21 сентября 2021 г.

В окончательной редакции 6 декабря 2021 г.

Принято к публикации 7 декабря 2021 г.

Предложен и экспериментально апробирован локальный метод микроволновой резонансной спектроскопии полупроводников. Микроволновый тракт спектрометра на базе зондовой станции Cascade Microtech оборудован коаксиальным резонатором специальной геометрии, за счет чего многократно повышена точность измерений в разработанном ранее методе вольт-импедансной спектроскопии. Разработана методика калибровки спектрометра и резонансных измерений комплексного импеданса системы зонд-образец. На нескольких дискретных частотах диапазона 50-250 MHz измерен импеданс тестовых структур с контактами Шоттки диаметром 30-60 $\mu$ m на монокристаллической пластине GaAs. Изучены нетривиальные резистивные свойства структур, заключающиеся в избыточном сопротивлении контакта, которое на 1-2 порядка превышает сопротивление растекания переменного тока в невозмущенной области полупроводника. Обнаруженный эффект предположительно связан с перезарядкой глубоких состояний в полупроводнике. Выполнен модельный расчет спектра импеданса, демонстрирующий согласие с экспериментальными спектрами.

Ключевые слова: микроволновый микроскоп, ближнее поле, зонд, резонатор, импеданс, полупроводник.

DOI: $10.21883 / J T F .2022 .03 .52145 .262-21$

\section{Введение}

Электромагнитная диагностика - эффективный инструмент исследования электрофизических характеристик полупроводниковых материалов и структур. Однако получение количественной информации на масштабах менее $100 \mu \mathrm{m}$ до сих пор остается серьезной проблемой. Высокочастотная (инфракрасная или оптическая) диагностика [1] не слишком информативна в отношении проводящих свойств, так как плазменная частота для полупроводников лежит в THz-диапазоне, пока недостаточно освоенном измерительной техникой. Кроме того, интерпретация оптических спектров коэффициентов отражения и прохождения осложняется межзонным поглощением, наличием примесных линий и фононных мод. Для полупроводниковых структур возникают дополнительные сложности из-за отражений на интерфейсах. Применение радиоволн [2] не обеспечивает требуемого разрешения даже для миллиметровых волн из-за фундаментального ограничения по критерию Рэлея. Уникальная (практически неограниченная) возможность локальных измерений появилась в связи с развитием в последние 30 лет методов ближнепольной микроволновой микроскопии (БММ). Для этой техники разрешение не ограничено длиной волны, а определяется размером апертуры зонда. Устройства низкочастотной части радиодиапазона $(f<50 \mathrm{MHz})$ не обладают достаточной импедансной чувствительностью, так как на масштабах менее $10-100 \mu \mathrm{m}$ приходится измерять емкость $C<1 \mathrm{fF}$. Предпочтителен микровол- новый (MB) диапазон, так как емкостной импеданс зонда $Z=-j /(2 \pi f C)$ уменьшается с ростом частоты. Чувствительность МВ микроскопов по емкости достигает $1 \mathrm{aF}$ [3]. Уже в начале периода бурного роста исследований по БММ (первая половина 90-х) разрешение устройств достигло $\sim 100 \mathrm{~nm}$ [4]. Первый коммерческий микроскоп с разрешением 3-5 в [5]. Разрешающая способность современных коммерческих микроскопов (Agilent/Keysight, PrimeNano) достигает 30-50 nm за счет адаптации к МВ устройствам зондов атомно-силовой микроскопии [6,7]. Революционный переход к атомарному разрешению $(\sim 0.2-0.3 \mathrm{~nm})$ совершен в [8] после реализации в БММ принципа туннельной микроскопии. Перспективность применения БММ для диагностики полупроводниковых материалов и структур в интересах твердотельной микро- и нано электроники в настоящее время не вызывает сомнений [9]. В соответствующих исследованиях применяются микроскопы как нанометрового [6,10-14], так и микронного разрешения [15-18]. С состоянием исследований по БММ можно ознакомиться по обзорам и монографиям [3,9,19-21].

Проблему получения при помощи БММ количественной информации об электрофизических характеристиках исследуемых объектов, в том числе структурированных, нельзя признать полностью решенной к настоящему времени. Для получения такой информации необходима теория, связывающая измеряемые характеристики с параметрами исследуемого объекта. Проблема заключается в сложной (зачастую не известной с достаточной точ- 
ностью) геометрии зондов микроскопов. Для построения аналитических моделей [22-28] используются упрощающие предположения, снижающие точность описания. Дополнительным осложняющим обстоятельством служит тот факт, что параметры моделей зависят от характеристик исследуемого объекта, т.е. должны быть заданы самосогласованно по данным калибровочных измерений $[15-18,29,30]$. Тем не менее в настоящее время при помощи классической БММ удается с достаточной степенью точности характеризовать простые объекты с одним подлежащим определению параметром, например, измерять удельную электропроводность объемно однородного материала, либо слоевое сопротивление пленочной структуры [11-18]. Для более сложных структур, содержащих несколько неизвестных параметров, характеристики объекта могут определяться путем решения обратной задачи, как правило, математически некорректной и нелинейной. Для успешного решения обратной задачи ключевым требованием является высокая точность теоретической модели. В работах $[31,32]$ обращено внимание на благоприятное обстоятельство: для зонда (антенны) правильной коаксиальной формы предложенная в [16] аналитическая модель становится достаточно точной для решения, как минимум, трехпараметрических обратных задач. Выполненная в [31] характеризация диодной структуры с разрешением 10-60 $\mu \mathrm{m}$ - пример решения указанной задачи. Отметим, что реализация соответствующих измерений требует применения специальных (неклассических) конструкций микроскопов.

Классический МВ микроскоп является частотноселективным прибором, так как имеет в своем составе резонатор. С появлением в последние 10-15 лет коммерческих зондовых станций, работающих в диапазоне до $\sim 100 \mathrm{GHz}$ (как, например, Cascade Microtech), появилась возможность построить МВ спектрометр, сочетающий в одном приборе локальность БММ с измерениями в широком частотном интервале. Предложенный в [33] и экспериментально апробированный в [32] метод вольт-импедансной $(\mathrm{Z}-\mathrm{V})$ спектроскопии полупроводников позволяет с микронным латеральным разрешением путем решения трехпараметрической обратной задачи получить полный набор электрофизических характеристик полупроводника: концентрацию, подвижность, тип свободных носителей заряда, удельную проводимость. В результате стало возможным перенести в МВ-диапазон такие информативные методы исследования полупроводников, как вольт-фарадную $(\mathrm{C}-\mathrm{V})$ диагностику [34] и адмиттансную спектроскопию (AC) [35-37]. Оба метода ранее применялись на низких (не более $10 \mathrm{MHz}$ ) частотах, из-за чего не обеспечивали микронное разрешение. Однако возможность получения $\mathrm{Z}-\mathrm{V}$-методом дополнительной информации о проводимости структур с барьером Шоттки осталась нереализованной в полном объеме в работе [32]. Проблема состояла в недостаточной точности измерения действительной части импеданса структур в низкочастотной $(f<500 \mathrm{MHz})$ области спектра. В настоящей работе предложено решение указанной проблемы и выполнено соответствующее исследование полупроводника.

\section{1. Точность измерения импеданса}

Разработанный в $[32,33] \mathrm{Z}-\mathrm{V}$-метод основан на измерении при помощи зондовой станции Cascade Microtech (CM) частотного спектра импеданса системы зонд-образец $Z(f, U)$ как функции приложенного к зонду постоянного напряжения $U$. Электрофизические параметры образца находятся путем решения соответствующей обратной задачи. При этом точность измерения импеданса $Z$ имеет решающее значение. Рассмотрим сначала реализованную в [32] классическую схему измерений, когда спектр $Z(f)$ определяется по спектру комплексного коэффициента отражения $S 11(f)=\Gamma(f)$ (в системе S-параметров) при непосредственном подключении СМ-зонда к одному из портов векторного анализатора цепей. Имеем

$$
\Gamma(f)=\frac{Z_{0}-Z(f)}{Z_{0}+Z(f)},
$$

где $Z_{0}$ - волновой импеданс подводящего кабеля. Пусть комплексный коэффициент отражения $\Gamma_{0}$ измеряется с некоторой погрешностью $\Gamma=\Gamma_{0}+\delta_{\Gamma}(1+i)$, т. е. полагаем ошибку $\delta_{\Gamma}$ измерения действительной и мнимой частей $\Gamma_{0}$ одинаковыми, причем $\delta_{\Gamma} \ll\left|\Gamma_{0}\right|$. В результате компоненты импеданса $Z=R-i X$ определяются с ошибкой $\delta_{R, X}$. В низкочастотной части спектра $Z(f)$ имеет место соотношение $X \gg R, Z_{0}$ [32] (см. также рис. 5), т.е. $\left|\Gamma_{0}\right| \approx 1$. Тогда из формулы (1) несложно получить

$$
\begin{gathered}
\frac{\delta_{X}}{X}=\frac{X}{2 Z_{0}} \delta_{\Gamma}, \\
\frac{\delta_{R}}{R}=\frac{X}{2 Z_{0}}\left(\frac{X}{R}\right) \delta_{\Gamma} .
\end{gathered}
$$

Можно видеть существенно более высокую погрешность определения сопротивления $\delta_{R} / R \gg \delta_{X} / X$. В измерениях [32] для диапазона $f=10-100 \mathrm{MHz}$ имели $X=10^{3}-10^{4} \Omega, R=5-50 \Omega$ (значения $R$ в работе [32] достоверно определены не были, но получены в данной pаботе (см. рис. $5, b)$ ). Тогда при $\delta_{\Gamma} \approx 10^{-3}, Z_{0}=50 \Omega$ получаем $\delta_{X} / X \approx 10^{-2}-10^{-1}, \delta_{R} / R \approx 2-20$, чем и обусловлена вышеуказанная проблема определения сопротивления $R$.

Рассмотрим теперь предлагаемую в данной работе схему измерений. Векторный анализатор цепей подключен к входу МВ-резонатора, как показано на рис. $1, b$. СМ-зонд присоединен к концу одного из плеч резонатора. В этом случае спектр коэффициента отражения 

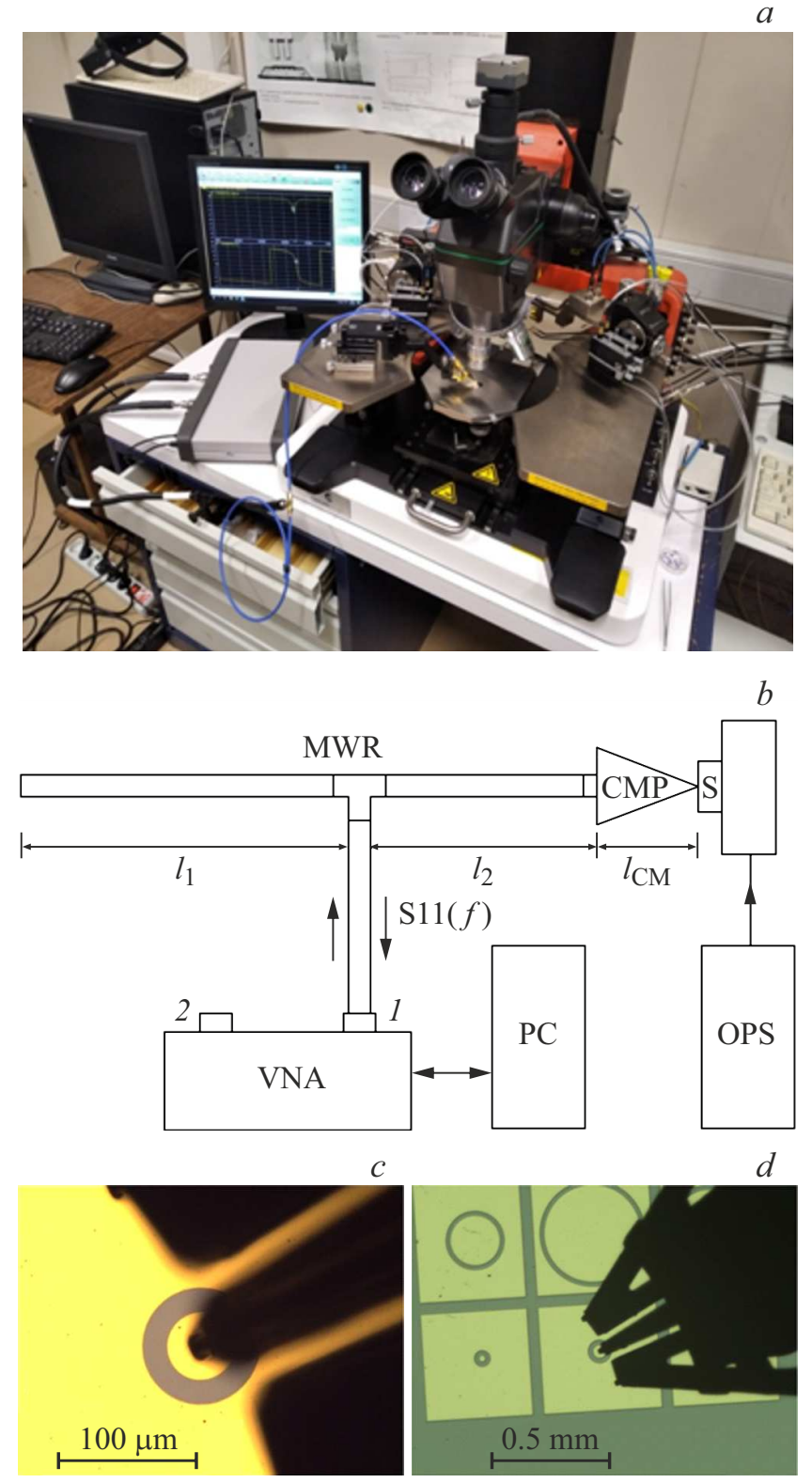

Рис. 1. $a-$ резонансный СМ-спектрометр; $b-$ блок-схема спектрометра. MWR - MB резонатор, CMP - CM-зонд, $\mathrm{S}$ - исследуемый образец, VNA - векторный анализатор цепей, РC - персональный компьютер, OPS - оптико-механическая система подведения; $c$ - подведенный к антенне S5 CM-зонд; $d-$ антенный модуль в контакте СМ-зонда с антенной S5.

представляется в виде [18]:

$$
\Gamma(f)=\frac{\frac{1-\beta}{Q_{0}}-2 i\left(\frac{f}{f_{0}}-1\right)}{\frac{1+\beta}{Q_{0}}+2 i\left(\frac{f}{f_{0}}-1\right)},
$$

где $f_{0}-$ резонансная частота, $Q_{0}-$ ненагруженная добротность, $\beta-$ параметр связи. Для модели резонатора в виде отрезка двухпроводной линии, показанной на рис. 2, $a$, параметры $f_{0}, Q_{0}, \beta$ по-
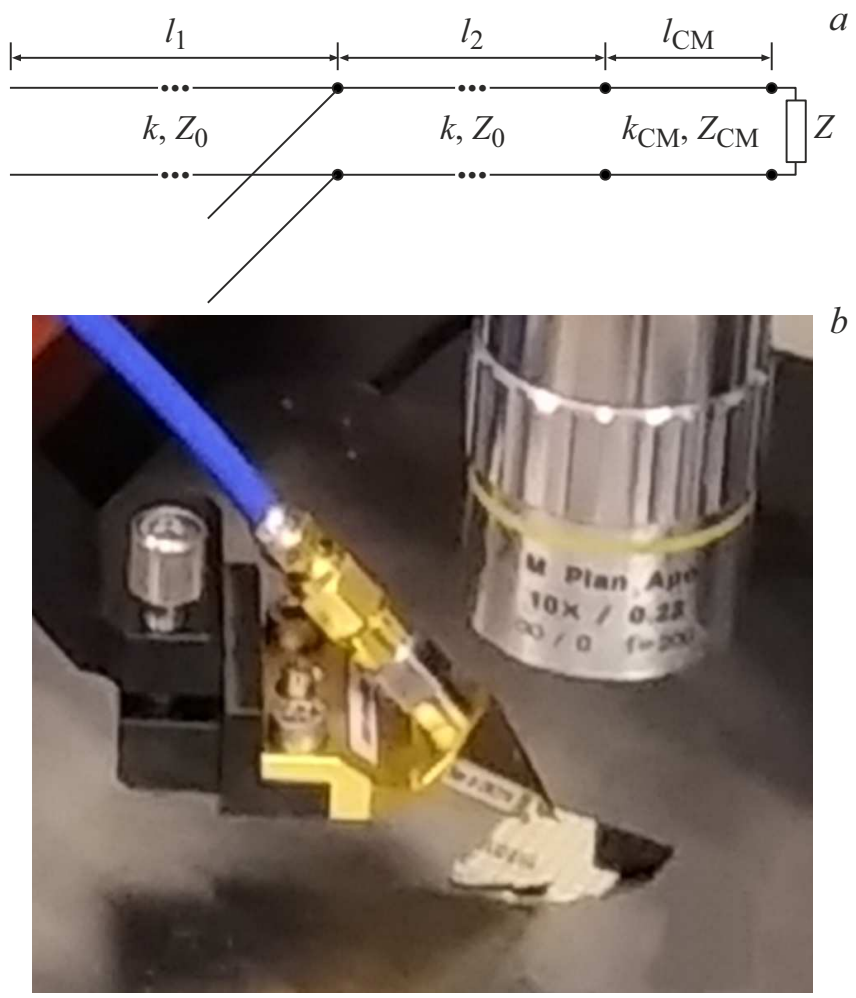

Рис. 2. $a$ - эквивалентная схема резонатора с СМ-зондом и

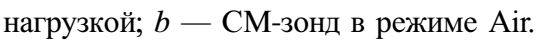

лучены в [18], если для упрощения анализа положить длину $l_{\mathrm{CM}}=0$. Имеем для полуволновой моды: $f_{0}=c /\left[2 l\left(1+Z_{0} /(\pi X)\right)\right], Q_{0}=Z_{0} / R_{\Sigma}, \beta=\rho^{2} /\left(Z_{0} R_{\Sigma}\right)$, $\rho=\pi Z_{0}\left(l_{1}-l_{2}\right) /(2 l), \quad R_{\Sigma}=\pi Z_{0} \delta+Z_{0}^{2} R / X^{2}$, где $c-$ скорость света, $\delta$ - параметр собственных потерь резонатора, введенный в разд. $3, l=l_{1}+l_{2}-$ полная длина резонатора $\left(l_{1}>l_{2}\right)$. Длины плеч резонатора $l_{1}, l_{2}$ и $\mathrm{CM-зонда} l_{\mathrm{CM}}$ показаны на рис. $1, b$. Тогда при учете погрешности измерения коэффициента Г получим ошибки измерения компонент импеданса:

$$
\begin{gathered}
\frac{\delta_{X}}{X}=\frac{X}{2 Z_{0}}\left[\frac{(1+\beta)^{2}}{\beta Q_{0}}\right] \delta_{\Gamma}, \\
\frac{\delta_{R}}{R}=\frac{X}{2 Z_{0}}\left(\frac{X}{R}\right)\left[\frac{(1+\beta)^{2}}{\beta Q_{0}}\right] \delta_{\Gamma} .
\end{gathered}
$$

Сравнивая выражения (2) и (4), видим, что использование резонаторной схемы измерений уменьшает погрешность определения импеданса в $\left(\beta Q_{0}\right) /(1+\beta)^{2}$ раз, что в нашем случае $\left(0.5<\beta<2, Q_{0} \approx 100\right)$ обеспечивает 10-20 кратный выигрыш в точности.

\section{2. Измерительная система и исследованный образец}

Спектрометр (рис. 1,a) содержал резонатор, два плеча которого, выполненные из отрезков полужесткого 
коаксиального кабеля RG-402, были подключены при помощи коаксиального тройного перехода SMA-KKK (розетка-розетка-розетка) к порту 1 векторного анализатора цепей (VNA) Anritsu MS46122B, как показано на рис. $1, b$. Калибровка VNA производилась при помощи SmartCal-модуля MN25208А для каждого из рабочих диапазонов: 40-70; 70-100; $140-180 ; 230-280 \mathrm{MHz}$. Управление работой VNA и регистрация отражения $\Gamma(f)$ осуществлялись персональным компьютером с помощью программного пакета ShockLine ${ }^{\mathrm{TM}}$. Нагрузкой одного из плеч спектрометра служил коаксиально-копланарный зонд (Z-probe coaxial) Z067-V3N-GSG-150 станции СМ. Изготовлено два резонатора R1, R2 на частоты: (R1) $f_{0} \approx 55$ и $160 \mathrm{MHz} ;(\mathrm{R} 2) f_{0} \approx 85$ и $160 \mathrm{MHz}$, отвечающие $\lambda / 2,3 \lambda / 2$ модам каждого. Длины плеч $l_{1,2}$ резонаторов были рассчитаны предварительно. Методика расчета описана в следующем разделе. Задача расчета (кроме того, чтобы получить частоту $f_{0}$ в нужных диапазонах) заключалась в том, чтобы параметр связи $\beta$ не выходил из диапазона $0.5-2$ как в отсутствие контакта зонда с образцом (режим Air), так и в контакте. В последнем случае предполагалось, что импеданс зонда
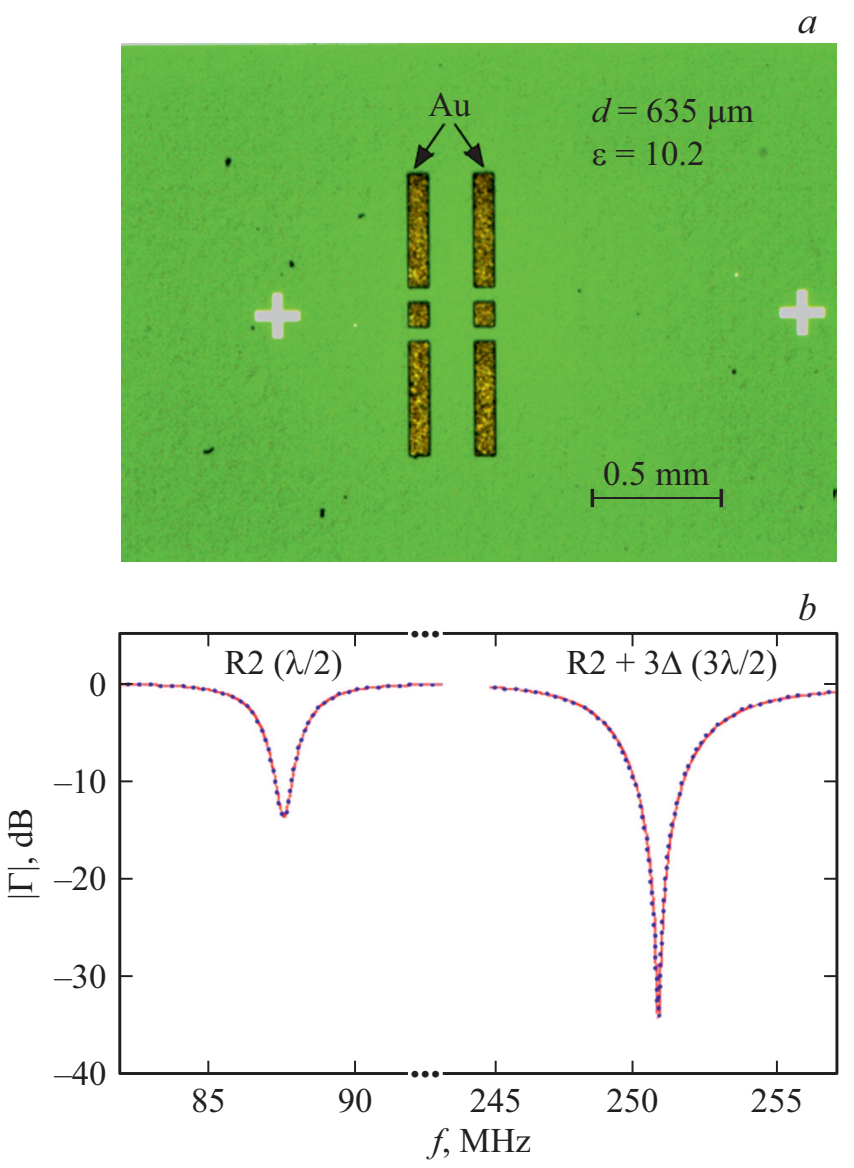

Рис. 3. $a-$ эталон импеданса $Z=\infty ; d$, $\varepsilon-$ толщина и диэлектрическая проницаемость подложки; $b-$ калибровочные спектры. Моды $\lambda / 2$ и $3 \lambda / 2$ резонатора $\mathrm{R} 2$ со вставкой $\Delta=0$ и $3 \Delta$. Сплошные линии - измерения в режиме Air, пунктирные линии - измерения эталона.

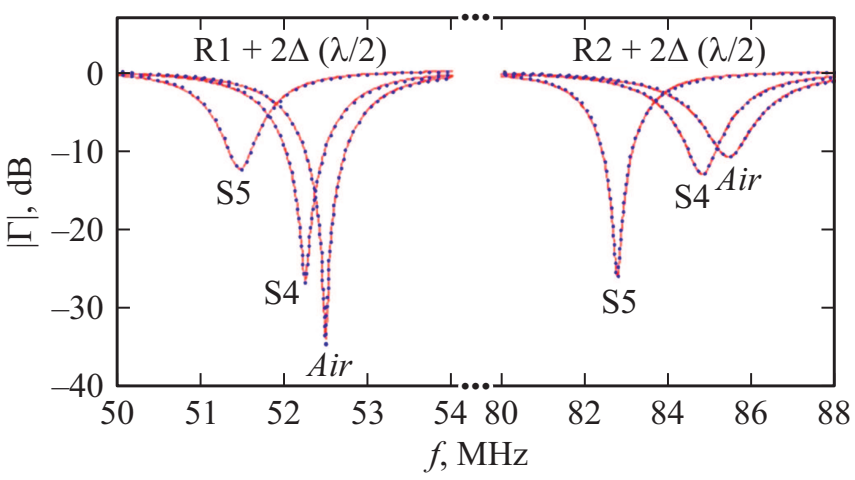

Рис. 4. Резонансные спектры в режиме калибровки (Air) и на антеннах S4, S5. Мода $\lambda / 2$ резонаторов R1 (слева), R2 (справа) со вставкой $2 \Delta$. Сплошные линии - измерения, пунктирные линии - расчет.

$Z\left(f_{0}\right)$ принимает полученные в работе [32] значения (для $R\left(f_{0}\right)$ выбирались ожидаемые значения). В указанном диапазоне $\beta$ реализуется хорошее согласование резонатора, когда $\left|\Gamma\left(f_{0}\right)\right|^{2}<-10 \mathrm{~dB}$, что обеспечивает достаточную точность определения импеданса $Z$. При согласовании, близком к идеальному, имеем $\beta \approx 1$, $\left|\Gamma\left(f_{0}\right)\right|<-40 \mathrm{~dB}$. В результате расчета и небольшого укорочения свободного плеча $l_{1}$ в процессе настроечных измерений получено: (R1) $l_{1}=110.8 \mathrm{~cm}, l_{2}=87.7 \mathrm{~cm}$; (R2) $l_{1}=66.5 \mathrm{~cm}, l_{2}=50.4 \mathrm{~cm}$. В измерениях образца производилось удлинение плеча $l_{1}$ на величину $\Delta$ и $2 \Delta$ $(\Delta \approx 1 \mathrm{~cm})$ при помощи вставок в виде SMА-разъемов вилка-розетка. Это обеспечивало по три независимых измерения $Z$ на $\lambda / 2$ моде каждого резонатора с незначительным сдвигом частоты $f_{0}$ при согласовании, не выходившем за указанный диапазон значений $\beta$. Требуемое согласование резонаторов по параметру $\beta$ на $3 \lambda / 2$ моде также достигалось за счет вставки $3 \Delta$ в плечо $l_{1}$. Примеры полученных резонансных кривых $|\Gamma(f)|$ в бесконтактном и контактном режимах представлены на рис. $3, b$ и 4 .

Исследовалась однородная монокристаллическая пластина GaAs толщиной $0.43 \mathrm{~mm}$, на поверхности которой была сформирована система концентрических контактов Шоттки (антенная система) в виде покрывающих всю поверхность модулей $1.5 \times 1.5 \mathrm{~mm}$, состоящих из девяти антенн разного диаметра. Этот же образец исследовался в [32]. В микроволновых измерениях мы использовали две антенны S4 и S5 (расположенные в одном из модулей в середине образца) с диаметром $a$ центрального контакта, равным 27 и $57 \mu \mathrm{m}$ соответственно. Контакт СМ-зонда с каждой антенной (рис. $1, c, d$ ) осуществлялся при помощи оптической системы подведения. Напряжение смещения между контактными площадками антенн $U=0$. При этом вблизи границы металл-полупроводник образуется обедненный слой толщины $d=77.5 \mathrm{~nm}$ при невозмущенной концентрации электронов $n_{0} \approx 2 \cdot 10^{17} \mathrm{~cm}^{-3}$, контактной разности по- 
тенциалов $U_{c}=0.75 \mathrm{~V}$ [32]. Измеряемый импеданс $Z\left(f_{0}\right)$ формируется за счет взаимодействия антенны со структурой, включающей обедненный слой полупроводника и нижележащую область с невозмущенной концентрацией. Как показано в [31], концентрическая антенна рассматриваемой геометрии является монополем, т.е. ее импеданс определяется только диаметром $a$ центрального контакта и не зависит от размеров внешней металлизированной площадки. Масштаб $a$ определяет также и разрешающую способность рассматриваемого метода. Зондирование образца производилось при низшей $\left(10^{-2} \mathrm{~mW}\right)$ мощности сигнала VNA, исключавшей нелинейные искажения $Z$.

\section{3. Методика измерений}

Задача заключалась в определении импеданса $Z\left(f_{0}\right)$ по измеренной резонансной характеристике $\Gamma(f)$ системы зонд-образец. С этой целью выполнялись расчеты с использованием эквивалентной схемы МВ-тракта спектрометра, показанной на рис. 2, $a$. В этой схеме резонатор и СМ-зонд (рис. 2, $b$ ) представлены в виде отрезков двухпроводных линий с волновыми импедансами $Z_{0}, Z_{\mathrm{CM}}$ и волновыми векторами $k, k_{\mathrm{CM}}$, причем $Z_{0}=Z_{\mathrm{CM}}=50 \Omega, k=k_{0} \sqrt{\varepsilon_{L}}\left(1+i \delta_{L}\right)$, $k_{\mathrm{CM}}=k_{0} \sqrt{\varepsilon_{\mathrm{CM}}}\left(1+i \delta_{\mathrm{CM}}\right), k_{0}=2 \pi f / c, \varepsilon_{L}, \delta_{L}-$ диэлектрическая проницаемость и собственные потери коаксиального кабеля, $\varepsilon_{\mathrm{CM}}, \delta_{\mathrm{CM}}-$ аналогичные параметры моделирующей зонд двухпроводной линии. Исследуемый образец рассматривался как нагрузка $Z$ на конце $\mathrm{CM}$-линии. Расчет резонансной характеристики производился по формулам

$$
\begin{gathered}
\Gamma(f)=\frac{Z_{0}-Z(f)}{Z_{0}+Z(f)}, \\
Z(f)=\frac{Z_{1}(f) Z_{2}(f)}{Z_{1}(f)+Z_{2}(f)}, \\
Z_{1}(f)=-i Z_{0} \operatorname{ctg}\left(k(f) l_{1}\right), \\
Z_{2}(f)=\frac{Z_{0}\left[Z_{\mathrm{CM}}^{i n}(f)+i Z_{0} \operatorname{tg}\left(k(f) l_{2}\right)\right]}{i Z_{\mathrm{CM}}^{i n}(f) \operatorname{tg}\left(k(f) l_{2}\right)+Z_{0}}, \\
Z_{\mathrm{CM}}^{i n}(f)=\frac{Z_{0}\left[Z+i Z_{0} \operatorname{tg}\left(k_{\mathrm{CM}}(f) l_{\mathrm{CM}}\right)\right]}{i Z \operatorname{tg}\left(k_{\mathrm{CM}}(f) l_{\mathrm{CM}}\right)+Z_{0}},
\end{gathered}
$$

где $l_{1}, l_{2}$ - длины образующих резонатор отрезков линий, а $l_{\mathrm{CM}}$ - длина моделирующей СМ-зонд линии (рис. $2, a$, а также рис. $1, b$ ). Отметим, что в формулах (7) частотная зависимость импедансов определяется набегом фазы на каждом отрезке линии, т. е. величиной $\sqrt{\varepsilon l}$, но не параметрами $\varepsilon, l$ в отдельности. Поэтому для коаксиального кабеля мы полагали $\varepsilon_{L}=2$ (фторопласт), а длины плеч $l_{1,2}$ определяли из измерений. Для СМ-зонда, наоборот, использовали геометрическую длину $l_{\mathrm{CM}}=4.5 \mathrm{~cm}$, а проницаемость $\varepsilon_{\mathrm{CM}}$ находили в эксперименте.
Импеданс образца $Z$ может быть найден только в том случае, когда параметры эквивалентной схемы на рис. 2, $a$ известны, поэтому было выполнено измерение при отключенном CM-зонде. Параметры образующей резонатор коаксиальной линии найдены из условия наилучшего соответствия измеренной и рассчитанной резонансных кривых путем минимизации невязки:

$$
N\left(l_{1}, l_{2}, \delta_{L}\right)=\int_{f_{a}}^{f_{b}}\left[\left|\Gamma_{e}(f)\right|-\left|\Gamma_{t}\left(f, l_{1}, l_{2}, \delta_{L}\right)\right|\right]^{2} d f,
$$

где $\Gamma_{e, t}-$ экспериментальная и теоретическая резонансные кривые. Расчет функции $\Gamma_{t}(f)$ по формулам (5)-(7) производится при $l_{\mathrm{CM}}=0$ (зонд отключен), $Z=\infty$ (разомкнутая линия). В этом случае формула (7b) имеет вид, аналогичный (7a) с заменой $l_{1} \rightarrow l_{2}$. Для поиска минимума невязки (8) использован программный пакет Mathcad:

$$
\mathbf{Y}=\left(\begin{array}{c}
Y_{1} \\
Y_{2} \\
Y_{3}
\end{array}\right)=\operatorname{Minimize}\left(N, l_{1}, l_{2}, \delta_{L}\right) .
$$

Рабочий диапазон частот $f_{a}-f_{b}$ в (8) определялся, как $\left|f_{a, b} / f_{0}-1\right|=1 / Q_{0}$, где резонансная частота и добротность резонатора $f_{0}, Q_{0}$ находились путем минимизации невязки $(8) N\left(f_{0}, Q_{0}, \beta\right)$, в которой в качестве $\Gamma_{t}\left(f, f_{0}, Q_{0}, \beta\right)$ использована функция (3). Отметим, что вычисленные длины $l_{1,2}$ оказались близкими к их геометрическим значениям. Для резонатора без вставки $(\Delta=0)$ длины $l_{1,2}$ приведены выше.

Измеряя резонансную характеристику $\Gamma_{e}(f)$ при подключенном СМ-зонде в отсутствие контакта с образцом (режим Air), определяли параметры $\varepsilon_{\mathrm{CM}}, \delta_{\mathrm{CM}}$ по минимуму невязки $(8)$, как функции $N\left(\varepsilon_{\mathrm{CM}}, \delta_{\mathrm{CM}}\right)$. При этом функция $\Gamma_{t}(f)$ рассчитывалась по формулам (5)-(7), где $Z=\infty$, а в качестве параметров $l_{1,2}, \delta_{L}$ использованы определенные на первом этапе значения. Примеры полученных значений параметров спектрометра представлены в таблице при $\Delta=0$. Как и ожидалось, СМ-зонд имеет заметно большие собственные потери $\delta_{\mathrm{CM}}$ по сравнению с потерями $\delta_{L}$ в коаксиальном кабеле. Тем не менее подключение зонда лишь незначительно (в пределах 10\%) уменьшает добротность резонатоpa $Q_{0}$, поскольку $l_{\mathrm{CM}} \ll l_{1,2}$.

Обратим внимание на важное обстоятельство, которое необходимо учитывать при поиске параметров модели спектрометра и импеданса $Z$ в (5)-(7) путем минимизации невязки (8). Функция $\left|\Gamma_{t}(f)\right|$ имеет одинаковый вид при двух различных наборах параметров формул (5)-(7). Это несложно понять, если записать спектр $\left|\Gamma_{t}(f)\right|$, используя представление (3). Получим одинаковые функции $\left|\Gamma_{t}(f)\right|$ при заменах $\beta \rightarrow 1 / \beta$, $Q_{0} \rightarrow \beta Q_{0}$. Однозначное решение (9) можно получить, если сравнивать фазы $\varphi_{e, t}(f)$ функций $\Gamma_{e, t}(f)$. Различный набор искомых параметров модели $(5)-(7)$ отвечает разным знакам производной фазы $d \varphi_{t}(f) /\left.d f\right|_{f=f_{0}}$ 
вблизи резонансной частоты $f_{0}$, что также легко видеть из формулы (3). Таким образом, если полученная в программе (9) и измеренная функции $\varphi_{e, t}(f)$ имеют разные знаки производной (т. е. найдено неверное решение), то необходимо изменить в программе (9) диапазон поиска решения, после чего придем к правильному набору параметров.

\section{4. Результаты исследований}

\section{1. Измерение импеданса}

Процесс измерения импеданса $Z$ сопровождался калибровкой, поскольку полученные параметры спектрометра немного изменяются в различных измерительных циклах в зависимости от качества сборки соединений, температуры и влажности в лаборатории. Для более точного определения $Z$ производились калибровочные измерения в начале и в конце каждого измерительного цикла. Калибровка может быть выполнена с использованием эталона импеданса $Z=\infty$ из набора стандартов $Z$ станции $\mathrm{CM}$ (рис. 3, $a$ ). Однако измерения функций $\Gamma(f)$ в контакте зонда с эталоном и в режиме Air показали идентичность полученных спектров, что можно видеть на рис. $3, b$. Поэтому применялся более простой измерительный процесс с калибровкой в режиме Air. Калибровка выполнялась только при подключенном СМ-зонде. При этом параметры зонда $\varepsilon_{\mathrm{CM}}, \delta_{\mathrm{CM}}$ считались фиксированными (см. таблицу), а уточнялись характеристики коаксиальных кабелей $l_{1}, l_{2}, \delta_{L}$. Измерения на рис. $3, b$ демонстрируют еще и тот факт, что в контакте СМ-зонда с металлическими поверхностями эталона не возникает паразитного импеданса. Аналогичный вывод можно распространить и на металлические поверхности антенн, т. е. найденный импеданс образца $Z$ характеризует контакт Шоттки металл-полупроводник.

Определение импеданса $Z=R-i X$ производилось по измерениям спектра $\Gamma_{e}(f)$ в контакте СМ-зонда с антеннами S4, S5 на поверхности полупроводниковой пластины (рис. $1, c, d$ ). Компоненты импеданса $R, X$ вычислялись путем минимизации невязки (8), как функции $N(R, X)$. При расчете спектров $\Gamma_{t}(f, R, X)$ в модели $(5)-(7)$ использовались значения параметров спектрометра, полученные в процессе калибровки. В (7c) полагали импеданс образца не зависящим от частоты $Z=Z\left(f_{0}\right)$, так как резонаторы $\mathrm{R} 1, \mathrm{R} 2$ имели достаточно высокую добротность $\left(Q_{0} \approx 100\right)$, а все расчеты

Параметры спектрометра

\begin{tabular}{c|c|c|c|c}
\hline$f_{0}, \mathrm{MHz}$ & 55 & 85 & 160 & 250 \\
\hline$\delta_{L} \cdot 10^{3}$ & 6.0 & 5.4 & 5.1 & 4.4 \\
\hline$\varepsilon_{\mathrm{CM}}$ & 1.05 & 1.06 & 1.19 & 1.11 \\
\hline$\delta_{\mathrm{CM}} \cdot 10^{3}$ & 8.6 & 7.3 & 9.6 & 10.2
\end{tabular}
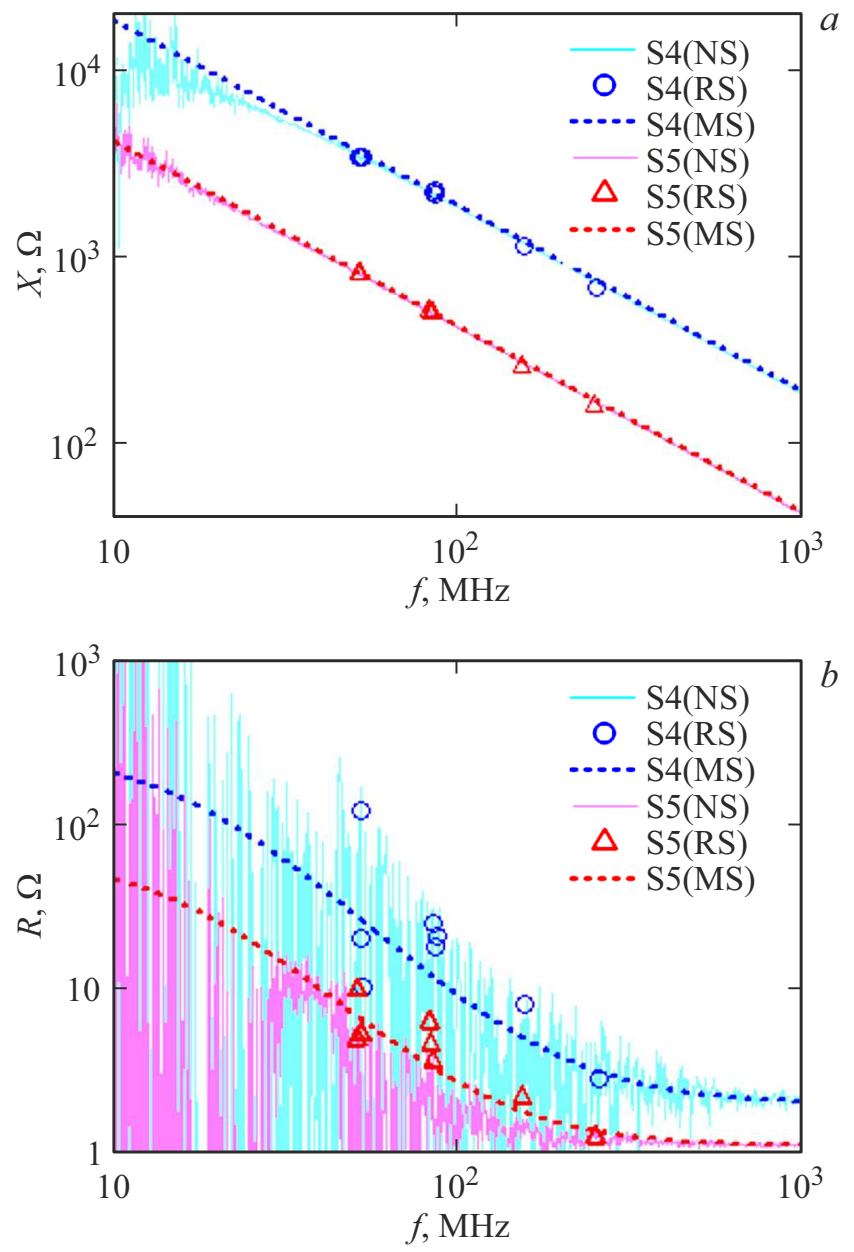

Рис. 5. Реактанс $(a)$ и сопротивление $(b)$ образца, полученные на антеннах S4, S5. NS - нерезонансная спектроскопия (сплошные линии), RS - резонансная спектроскопия (значки), MS - модельные спектры (штриховые линии).

делались в узком диапазоне вблизи резонансной частоты $\left|f / f_{0}-1\right| \leq 1 / Q_{0}$. Примеры полученных спектров представлены на рис. 4. Можно видеть очень хорошее соответствие расчетов и измерений после подстановки в теоретическую модель (5)-(7) найденных параметров спектрометра и импеданса образца. Видна также достаточно высокая информативность спектров $\Gamma(f)$ к характеристикам контакта Шоттки для обеих антенн.

Результаты измерения реактанса $X$ и сопротивления $R$ представлены на рис. 5. Приведены зависимости $X(f)$, $R(f)$, полученные методами нерезонансной (NS) [32] и резонансной (RS) спектроскопий. Небольшое различие резонансных частот для $\lambda / 2$-моды каждого резонатора, отвечающее трем значениям $\Delta$, на рис. 5 практически не отображается. На рис. 5, $a$ можно видеть очень хорошее соответствие спектров $X(f)$, измеренных обоими методами, что свидетельствует о достоверности полученных данных. Отметим также незначительный разброс значений $X(f)$ от точки к точке (более $10^{3}$ дискретных частот в NS-измерениях) в диапазоне $f>20-30 \mathrm{MHz}$, 
что и прогнозировалось в выполненном в разд. 1 анализе. Иная картина наблюдается для спектров $R(f)$ (рис. 5, $b$ ). Низкочастотная часть NS-спектров $(f<200-300 \mathrm{MHz})$ сильно зашумлена. Более того, в области низких частот величина $R$ на отдельных частотах принимает отрицательные значения, поскольку из-за шумов NS-спектрометра измеряемый коэффициент отражения $|\Gamma|=\left|\Gamma_{0}\right|+\delta_{\Gamma}$ может иметь значения $|\Gamma|>1$, так как $\left|\Gamma_{0}\right| \approx 1$ при $X \gg Z_{0}=50 \Omega$. В результате NS-спектры $R(f)$ на низких частотах в линейном масштабе выглядят практически симметричными относительно уровня $R=0$. На рис. 5, $b$ спектры $R(f)$ показаны в логарифмическом масштабе, в котором значения $R<0$ не отображаются.

Таким образом, NS не позволяет достоверно судить о сопротивлении зонда $R$ в низкочастотной части спектра, причем разброс данных резко увеличивается с уменьшением диаметра антенны. Напротив, RS дает существенно более стабильные значения $R$. На рис. $5, b$ отчетливо видно превышение $\mathrm{R}$ (до двух порядков) над высокочастотным значением $r_{0}=1-2 \Omega$. RS-измерения демонстрируют монотонный рост сопротивления с понижением частоты, из-за которого $R$ достигает $10-100 \Omega$ на низших частотах спектрометра. С уменьшением диаметра $a$ контакта наблюдается более резкий рост $R$. Отметим, что полученные в [32] недостоверные данные об избыточном сопротивлении $R$ не препятствовали решению задачи той работы - определению основных электрофизических характеристик полупроводника. Низкочастотная часть спектра $R(f)$ несет в себе важную дополнительную информацию о проводящих свойствах контакта Шоттки.

\section{2. Глубокие состояния}

Обнаруженное избыточное сопротивление $R>r_{0}$ в диапазоне $10-250 \mathrm{MHz}$ не объясняется элементарной теорией контакта Шоттки [38,39]. Согласно этой теории, импеданс контакта $Z$ описывается эквивалентной схемой, показанной на рис. $6, a$, где

$$
R_{c}=\frac{r_{c}}{S_{a}}, \quad C_{d}=\frac{\varepsilon_{0} \varepsilon^{\prime} S_{a}}{d}, \quad r_{0} \approx \frac{1}{2 \sigma_{0} a} .
$$

Здесь $d(U=0)=77.5 \mathrm{~nm}-$ толщина обедненного слоя при нулевом напряжении смещения [32], $S_{a}=\pi a^{2} / 4$ - площадь контакта, $\varepsilon_{0}-$ электрическая постоянная вакуума, $\varepsilon^{\prime}=12.9-$ диэлектрическая проницаемость $\mathrm{GaAs}, \sigma_{0}-$ проводимость невозмущенной области полупроводника. Сопротивление $r_{c}=5 \cdot 10^{4} \Omega \cdot \mathrm{cm}^{2}$ определено нами по измерению на постоянном токе. В диапазоне $f>1 \mathrm{MHz}$ имеем $R_{c} \gg 1 /\left(\omega C_{d}\right)(\omega=2 \pi f)$, в результате чего эквивалентное последовательное сопротивление $1 /\left(R_{c}\left(\omega C_{d}\right)^{2}\right) \ll r_{0}=1-2 \Omega$ при $f>10 \mathrm{MHz}$, $\sigma_{0} \approx 84(\Omega \cdot \mathrm{cm})^{-1}$ [32]. Поэтому в рассматриваемом частотном диапазоне можно пренебречь влиянием $R_{c}$

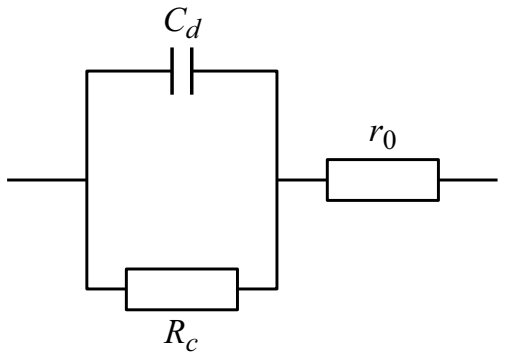

$a$

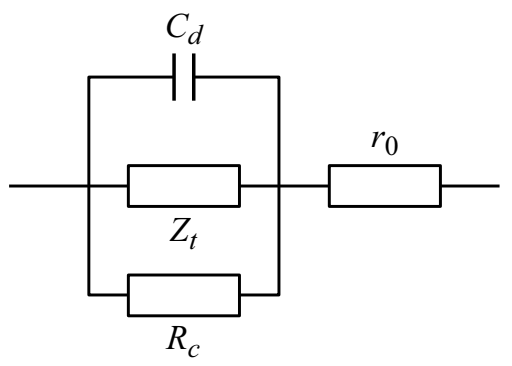

$b$

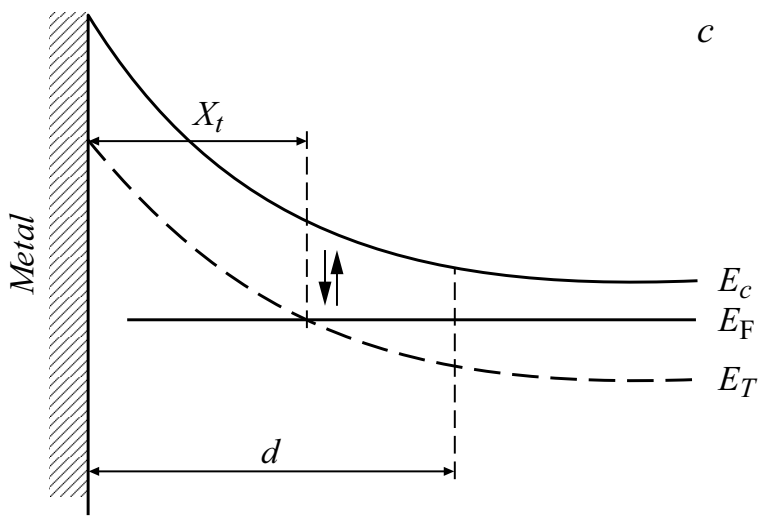

Рис. 6. $a-$ эквивалентная схема контакта Шоттки; $b-$ эквивалентная схема с учетом глубоких состояний; $c-$ уровни энергии контакта Шоттки с глубокими состояниями. $E_{\mathrm{F}}-$ уровень Ферми, $E_{c}-$ уровень легирующей примеси, $E_{T}$ - глубокий уровень.

на импеданс контакта. Производя вычисления по эквивалентной схеме, изображенной на рис. $6, a$, для антенн $\mathrm{S} 4$, S5 получаем MS зависимости $X(f)$, показанные на рис. 5, $a$ пунктирными линиями. При этом расчет дает постоянное сопротивление $R(f>10 \mathrm{MHz}) \approx r_{0}$, представляющее собой сопротивление растекания переменного тока в невозмущенной области полупроводника под обедненным слоем. Выполненные измерения, напротив, дали $R \gg r_{0}$ в низкочастотной части спектра.

Избыточное сопротивление в спектре $R(f)$ может объясняться присутствием в полупроводнике глубоких состояний (ловушек), связанных с примесями или дефектами [40]. В барьере Шоттки уровень Ферми $E_{\mathrm{F}}$ пересекает глубокий уровень $E_{T}$ на некотором расстоянии $x_{t}$ от границы металл-полупроводник, как показано на рис. 6,c. В приближении полного обеднения легко получить

$$
\frac{x_{t}}{d}=1-\sqrt{\frac{E_{\mathrm{F}}-E_{T}}{U_{\mathrm{c}}}} .
$$


Переменное напряжение приводит к колебаниям положения уровня Ферми, из-за чего происходит перезарядка глубоких уровней. Основной вклад в перезарядку вносят ловушки из тонкого $(\sim 10 \mathrm{~nm})$ слоя полупроводника, расположенного вблизи $x_{t}$. Вследствие перезарядки ловушек эквивалентная схема контакта трансформируется за счет дополнительного комплексного импеданса $Z_{t}[37]$ (рис. $6, b$ ), определяемого последовательным соединением соответствующих емкости и сопротивления. Согласно [37], имеем

$$
\begin{gathered}
Z_{t}=\frac{1}{\Delta C S_{a}}\left(\frac{-i}{\omega}+\tau\right), \\
\Delta C=\frac{\varepsilon_{0} \varepsilon^{\prime}}{d} \frac{n_{t}}{n_{0}} \frac{1-\frac{x_{t}}{d}}{1+\frac{x_{t}}{d} \frac{n_{t}}{n_{0}}} .
\end{gathered}
$$

Здесь $\tau-$ время релаксации заполнения ловушек, $n_{t}-$ концентрация ловушек. Результаты расчета спектра импеданса $Z(f)$ по эквивалентной схеме на рис. $6, b$ показаны на рис. 5 модельными спектрами MS. В расчетах приняты следующие значения параметров ловушек: $\tau=10^{-8} \mathrm{~s}, n_{t} / n_{0}=5 \cdot 10^{-2}$, $x_{t} / d=0.5$ при глубине их уровня $E_{\mathrm{F}}-E_{T}=0.2 \mathrm{eV}$. Можно видеть, что модельные спектры $R(f)$ на рис. $5, b$ неплохо соответствуют экспериментальным данным. С учетом (12), (13) в схеме на рис. 6, $b$ получим $C_{d} \leq \operatorname{Im}\left[Z^{-1}(\omega)\right] / \omega \leq C_{d}+\Delta C S_{a}$. В рассматриваемом случае имеем соотношение $\Delta C S_{a} \ll C_{d}$. В результате емкостная составляющая импеданса $Z_{t}$ практически не влияет на спектр $X(f)$, из-за чего кривые MS на рис. $5, a$, рассчитанные по эквивалентным схемам на рис. $6, a, b$, совпадают.

Параметры глубоких состояний обычно исследуются методом АС в диапазоне частот $f<10 \mathrm{MHz}$ при размере контакта $a>100 \mu \mathrm{m}[37,41-44]$. С этой целью специально создается контакт с барьером Шоттки. Исследование ловушек с полученными здесь характеристиками затруднительно посредством классической $\mathrm{AC}$ при комнатной температуре, так как при макроскопическом контакте их сопротивление маскируется сопротивлениями обедненного слоя $R_{c}$ и растекания $r_{0}$. Действительно, используя эквивалентную схему на рис. $6, b$, импеданс контакта можно представить, как

$$
Z=-\frac{i}{\omega C_{d}}+\frac{R_{t} f(\omega)}{1+\omega^{2} \tau_{d}^{2} f^{2}(\omega)}+r_{0}
$$

где $R_{t}=\tau /\left(\Delta C S_{a}\right), \quad f(\omega)=\left(1+\omega^{2} \tau^{2}\right) / \omega^{2} \tau^{2}$. Формула (14) дает асимптотики спектра сопротивления: низкочастотную $(\omega \tau \ll 1) R=R_{S}=R_{t}\left(\tau / \tau_{d}\right)^{2}+r_{0}$ и высокочастотную $(\omega \tau \gg 1) R=R_{t} /\left(\omega \tau_{d}\right)^{2}+r_{0}$, где $\tau_{d}=R_{t} C_{d}$. Для принятых в расчетах значений параметров имеем сопротивление на единицу площади контакта $r_{t}=R_{t} S_{a}=2.8 \Omega \cdot \mathrm{cm}^{2}$ при $\tau_{d}=4.2 \cdot 10^{-7} \mathrm{~s}$. Тогда для контактов S4 и S5 получим низкочастотное сопротивление насыщения $R_{S}=281$ и $63 \Omega$ соответственно, т.е. $R_{S} \gg r_{0} \approx 2$ и $1 \Omega$. Насыщение функции $R(f)$ достигается при $f \approx 3-5 \mathrm{MHz}$, поэтому на рис. $5, b$ видна только тенденция к насыщению. С понижением частоты в диапазоне $f<1 \mathrm{MHz}$ начинается монотонный рост функции $R(f)$ под влиянием сопротивления $R_{c}$, которым мы пренебрегли в формуле (14). В диапазоне $f>50 \mathrm{MHz}$ имеем высокочастотную (убывающую $\propto f^{-2}$ ) зависимость избыточного сопротивления, так что при $f>250 \mathrm{MHz}$ получаем $R \approx r_{0}$. Таким образом, полученный эффект состоит в том, что в диапазоне частот $10 \mathrm{MHz}<f<200 \mathrm{MHz}$ имеем $r_{0}<R<R_{S}$ при $R_{S} \gg r_{0}$. Указанный эффект отчетливо наблюдается только при достаточно малом диаметре контакта $a<100 \mu \mathrm{m}$, так как $R_{S} \sim a^{-2}$, тогда как $r_{0} \sim a^{-1}$, т.е. отношение $R_{S} / r_{0} \sim a^{-1}$. Функция $\operatorname{Re}\left(Z(\omega)^{-1}\right) / \omega$ имеет максимум, который, как несложно получить при помощи формулы (14), реализуется при $\omega \tau=1$ и связан с наличием избыточного сопротивления контакта. Измерение этого максимума, исчезающего с увеличением диаметра $a$, дает наиболее простой способ определения времени перезарядки ловушек $\tau$ [37]. В рассматриваемой модели исследованного образца максимум реализуется на частоте $f=16 \mathrm{MHz}$. Учитывая названные трудности исследования ловушек при низкочастотных измерениях адмиттанса макроскопических контактов, в классической $\mathrm{AC}$ делается проход по температуре, начиная от температуры жидкого гелия. При низких температуpax увеличивается время перезарядки ловушек $\tau$, что делает возможным измерить связанное с ловушками изменение емкости контакта на низких частотах. Таким образом, разработанный нами NS- RS-метод, фактически являющийся MB-аналогом классической $\mathrm{AC}$, не только обеспечивает локальность измерений, но и позволяет определять параметры ловушек при комнатной температуре.

Введем эффективную проводимость обедненного слоя $\sigma_{t}=d / r_{t}$. В рассматриваемом случае получаем $\sigma_{t}=2.8 \cdot 10^{-6}(\Omega \cdot \mathrm{cm})^{-1}$. Именно проводимость $\sigma_{t}$ была определена в [32] из решения трехпараметрической обратной задачи с использованием неточно измеренного низкочастотного спектра $R(f)$. Тем не менее для антенн S4, S5 в [32] мы получили $\sigma_{t}=(1.4,3.2) \cdot 10^{-6}(\Omega \cdot \mathrm{cm})^{-1}$ при $U=0-$ значения, неплохо соответствующие результату данной работы. Заметим, что в рассматриваемой модели ловушек, согласно (12), имеем комплексную проводимость обедненного слоя $\tilde{\sigma}_{t}=i \sigma_{t} \omega \tau /(1+i \omega \tau)$, из-за чего обратная задача становится четырехпараметрической с дополнительно определяемым параметром $\tau$.

Отметим также, что обнаруженное в настоящей работе избыточное сопротивление $R(f)>r_{0}$ определяет джоулевы потери в барьерном контакте в некотором частотном интервале $(10-250 \mathrm{MHz}$ в нашем случае). Данное наблюдение может быть интересно для ряда приборных применений высокочастотных диодов Шоттки микронного и субмикронного диаметра, в частности, смесителей. Например, для $a=0.5 \mu \mathrm{m}$, используя эквивалентную схему на рис. $6, b$, в диапазоне $f \approx 1 \mathrm{GHz}$ 
нетрудно получить $R \approx 600 \Omega>r_{0} \approx 100 \Omega$, т. е. потери на разностной частоте смесителя на основе исследованного материала обусловлены в основном избыточным сопротивлением.

\section{Заключение}

В работе развит метод микроволновой резонансной спектроскопии полупроводников, повышающий потенциальные возможности нерезонансной Z-V-спектроскопии, разработанной в [32,33]. Задача Z-V-спектроскопии, заключающаяся в определении локальных значений основных электрофизических характеристик полупроводника, успешно решается при помощи NS-измерений в микроволновом диапазоне. Получение дополнительной информации о резистивных свойствах контакта Шоттки потребовало изменения техники измерений. Именно этой цели служит разработанный здесь RS-метод. Метод позволил с латеральным разрешением 30-60 $\mu \mathrm{m}$ исследовать спектр сопротивления полупроводниковой структуры с барьером Шоттки. Наблюдаемое в диапазоне $50-250 \mathrm{MHz}$ избыточное сопротивление контакта Шоттки, весьма вероятно, связано с наличием глубоких состояний в исследованном материале. Для подтверждения выдвинутой гипотезы целесообразно провести дополнительные исследования. Возможности разработанной RS- и NS-техники на базе зондовой станции СМ позволяют выполнить измерения для контактов меньшего диаметра, вплоть до $a \sim 3-5 \mu \mathrm{m}$, в частотном диапазоне до 20-30 GHz и более. Целесообразно увеличить количество рабочих частот $\mathrm{RS}$ в информативной части спектра $R(f)$. Интересно также произвести параллельные измерения независимыми методами, например, методом нестационарной спектроскопии глубоких уровней.

Сочетание NS- и RS-измерений дает экспериментальный материал в виде спектров $R(f), X(f)$, необходимый для решения обратных задач характеризации полупроводников. В работах $[31,32]$ продемонстрирована возможность решения трехпараметрических обратных задач. Применение коаксиальных антенн обеспечивает необходимую точность аналитической модели, используемой для их решения. Дальнейшее развитие $\mathrm{Z}-\mathrm{V}$-спектроскопии видится в ее приложении к планарным полупроводниковым структурам. Отметим, что даже для однородного по глубине материала применение $\mathrm{Z}-\mathrm{V}$-метода связано с исследованием структуры в виде обедненного слоя над невозмущенной областью полупроводника. Благодаря созданию такой структуры удается раздельно определить концентрацию и подвижность носителей заряда. Третьим определяемым параметром является проводимость обедненного слоя. Для более сложных структур (например, системы пленка-подложка) Z-V-техника потребует решения обратной задачи с числом определяемых параметров более трех. В силу математической некорректности задачи возможности подобной диагностики нуждаются в отдельном исследовании.

В нынешней реализации $\mathrm{Z}-\mathrm{V}$-спектроскопия является неразрушающим, но контактным методом. Сформированная на поверхности образца антенная система после выполнения измерений может быть удалена путем несложных технологических операций. Аналогичные бесконтактные измерения требуют разработки специальных микроантенн. Принципиально сохранить правильную геометрическую форму бесконтактной антенны, в частности, примененную нами концентрическую форму. Указанное требование продиктовано необходимостью решения обратной задачи, как составной части разработанного метода. $\mathrm{C}-\mathrm{V}$-диагностика полупроводников с использованием классических БММ нанометрового разрешения осуществлена в работах $[6,11,12,14]$. Выполненные в этих работах измерения не позволили получить набор электрофизических параметров исследовавшихся образцов в объеме, достигнутом в [31,32]. Одна (но не единственная) из причин возникших трудностей видится в сложной геометрии существующих нанометровых зондов, не позволяющей выполнить достаточно аккуратное моделирование. Примененная в работе техника СМ-измерений, скорее всего, позволит довести разрешающую способность спектрометра до 3-5 $\mu \mathrm{m}$. Вопрос о возможности продвижения к нанометровому разрешению пока остается открытым. Для раздельного определения концентрации и подвижности носителей принципиально важно разработать технику измерения не только мнимой (емкости $C$ ), но также и действительной (сопротивления $R$ ) части импеданса $Z$. Последнее важно и для исследования проводящих свойств обедненного слоя, выполненного в настоящей работе.

Еще раз подчеркнем значение аналитических моделей электродинамики системы зонд-образец. В ряде работ исследования потенциальных возможностей БММ (разрешающей способности, чувствительности и пр.) производятся на основе компьютерного моделирования методами конечных элементов [45-50]. При этом используются собственные или коммерческие программы электромагнитной симуляции. Иногда на основе соответствующих вычислений осуществляется количественная характеризация образцов достаточно простой структуры [3,48-50]. Принципиальным недостатком указанного подхода является длительное время решения прямой задачи. Так, в работе [47] для БММ определенной конфигурации и образца с заданной диэлектрической проницаемостью время расчета резонансной характеристики составило несколько часов. При переходе к обратным задачам (в особенности для сложных структур) время вычислений является ключевым параметром. Алгоритмы решения таких задач используют итерационный спуск с пошаговым решением прямой задачи. По нашему опыту, даже для простых объектов требуется несколько сотен итераций (часто тысяч). В результате определение характеристик исследуемого объекта становится практически невозможным в реальном времени. 
Предпринимаемые усилия по сокращению временных затрат [51] пока не привели к существенному улучшению ситуации. Кроме того, моделирование формы зонда БММ является отдельной проблемой даже при наличии его микрофотографий в нескольких проекциях. Как правило, определение геометрических параметров модели требует дополнительных калибровочных измерений, что еще больше увеличивает время вычислений. По нашему мнению, только аналитические модели зондов на сегодня позволяют строить эффективные алгоритмы решения обратных задач ближнепольной микроскопии.

\section{Финансирование работы}

Использовано оборудование Центра коллективного пользования ИФМ РАН „Физика и технология микрои наноструктур“.

\section{Конфликт интересов}

Авторы заявляют, что у них нет конфликта интересов.

\section{Список литературы}

[1] J.F. Power. Rev. Sci. Instrum., 73, 4057 (2002). DOI: $10.1063 / 1.1517054$

[2] J. Krupka. Meas. Sci. Technol., 24, 062001 (2013). DOI: $10.1088 / 0957-0233 / 24 / 6 / 062001$

[3] K. Lai, W. Kundhikanjana, M.A. Kelly, Z.-X. Shen. Appl. Nanosci., 1, 13 (2011). DOI: 10.1007/s13204-011-0002-7

[4] C. Gao, T. Wei, F. Duewer, Y. Lu, X.-D. Xiang. Appl. Phys. Lett., 71, 1872 (1997).

[5] V.V. Talanov, A. Scherz, R.L. Moreland, A.R. Schwartz. Appl. Phys. Lett., 88, 134106 (2006). DOI: 10.1063/1.2189147

[6] H.P. Huber, I. Humer, M. Hochleitner, M. Fenner, M. Moertelmaier, C. Rankl, A. Imtiaz, T.M. Wallis, H. Tanbakuchi, P. Hinterdorfer, P. Kabos, J. Smoliner, J.J. Kopanski, F. Keinberger. J. Appl. Phys., 111, 014301 (2012). DOI: $10.1063 / 1.3672445$

[7] A. Tselev, N.V. Lavrik, I. Vlassiouk, D.P. Briggs, M. Rutgers, R. Proksh, S.V. Kalinin. Nanotechnology, 23, 385706 (2012). DOI: $10.1088 / 0957-4484 / 23 / 38 / 385706$

[8] J. Lee, C.J. Long, H. Yang, X.-D. Xiang, I. Takeuchi. Appl. Phys. Lett., 97, 18311 (2010). DOI: 10.1063/1.3514243

[9] S. Berweger, T.M. Wallis, P. Kabos. IEEE Micrwave Mag., 21, 36 (2020). DOI: 10.1109/MMM.2020.3008305

[10] G. Gramse, M. Kasper, L. Fumagalli, G. Gomila, P. Hinterdorfer, F. Kienberger. Nanotechnology, 25, 145703 (2014). DOI: $10.1088 / 0957-4484 / 14 / 38 / 145703$

[11] O. Amster, F. Stanke, S. Friedman, Y. Yang, St.J. Dixon-Warren, B. Drevniok. Microelectron. Reliability, 76-77, 214 (2017). DOI: 10.1016/j.microrel.2017.07.082

[12] S. Hommel, N. Killat, A. Altes, T. Schweinboeck, F. Kreupl. Microelectron. Reliability, 76-77, 221 (2017). DOI: 10.1016/j.microrel.2017.06.050

[13] S. Berweger, G.A. MacDonald, M. Yang, K.J. Coakley, J.J. Berry, K. Zhu, F.W. DelRio, T.M. Wallis, P. Kabos. NanoLett., 17, 1796 (2017).

DOI: 10.1021/acs.nanolett.6b05119
[14] A. Buchter, J. Hoffman, A. Delvallee, E. Brinciotti, D. Hapiuk, C. Licitra, K. Louarn, A. Arnoult, G. Almuneau, F. Piquemal, M. Zeier, F. Kienberger. Rev. Sci. Instrum., 89, 023704 (2018). DOI: $10.1063 / 1.5015966$.

[15] A.N. Reznik, E.V. Demidov. J. Appl. Phys., 113, 094501 (2013). DOI: 10.1063/1.4794003

[16] A.N. Reznik, S.A. Korolyov. J. Appl. Phys., 119, 094504 (2016). DOI: $10.1063 / 1.4943068$

[17] A.N. Reznik, S.A. Korolyov, M.N. Drozdov. J. Appl. Phys., 121, 164503 (2017). DOI: 10.1063/1.4982676

[18] S.A. Korolyov, A.N. Reznik. Rev. Sci. Instrum., 89, 023706 (2018). DOI: $10.1063 / 1.5013113$

[19] B.T. Rosner, D.W. Van der Weide. Rev. Sci. Instrum., 73, 2505 (2003). DOI: $10.1063 / 1.1482150$

[20] S.M. Anlage, V.V. Talanov, A.R. Schwartz. Principles of Near-Field Microwave microscopy", in Scanning Probe Microscopy: Electrical and Electromechanical Phenomena at the Nanoscale, ed. by S. Kalinin, A. Gruverman (Springer Verlag, Berlin, 2007), p. 215-253.

[21] A. Imtiaz, T.M. Wallis, P. Kabos. IEEE Micrwave Mag., 15, 52 (2014). DOI: 10.1109/MMM.2013.2288711

[22] D.E. Steinhauer, C.P. Vlahacos, S.K. Dutta, F.C. Wellstood, S.M. Anlage. Appl. Phys. Lett., 71, 1736 (1997).

[23] C. Gao, X.-D. Xiang. Rev. Sci. Instrum., 69, 3846 (1998).

[24] C. Gao, B. Hu, P. Zhang, M. Huang, W. Liu, I. Takeuchi. Appl. Phys. Lett., 84, 4647 (2004). DOI: 10.1063/1.1759389

[25] A.N. Reznik, N.V. Yurasova. J. Appl. Phys., 98, 114701 (2005). DOI: 10.1063/1.2138798

[26] Z. Wang, M.A. Kelly, Z.-X. Shen, L. Shao, W.-K. Chu, H. Edwards. Appl. Phys. Lett., 86, 153118 (2005). DOI: $10.1063 / 1.1891296$

[27] A. Imtiaz, S.M. Anlage. J. Appl. Phys., 100, 044304 (2006). DOI: $10.1063 / 1.2234801$

[28] T. Nozokido, M. Ishido, R. Seto, J. Bae. J. Appl. Phys., 118, 114905 (2015). DOI: 10.1063/1.4931149

[29] A. Imtiaz, T. Baldwin, H.T. Nembach, T.M. Wallis, P. Kabos. Appl. Phys. Lett., 90, 243105 (2007). DOI: $10.1063 / 1.2748307$

[30] A.N. Reznik, V.V. Talanov. Rev. Sci. Instrum., 79, 113708 (2008). DOI: 10.1063/1.3020705

[31] A.N. Reznik, N.V. Vostokov, N.K. Vdovicheva, S.A. Korolyov, V.I. Shashkin. J. Appl. Phys., 122, 244505 (2017). DOI: $10.1063 / 1.4995330$

[32] А.Н. Резник, Н.В. Востоков, Н.К. Вдовичева, В.И. Шашкин. ЖТФ, 90 (11), 1944 (2020). DOI: 10.21883/JTF.2020.11.49988.150-20 [A.N. Reznik, N.V. Vostokov, N.K. Vdovicheva, V.I. Shashkin. Tech. Phys., 64 (11), 1859 (2020). DOI: 10.1134/S1063784220110237]

[33] А.Н. Резник, Н.К. Вдовичева. ЖТФ, 89 (11), 1813 (2019). DOI: $10.21883 /$ JTF.2019.11.48350.150-19 [A.N. Reznik, N.K. Vdovicheva. Tech. Phys., $64(11), 1722$ (2019). DOI: $10.1134 / \mathrm{S} 1063784219110240]$

[34] D.K. Schroder. Semiconductor Material and Device Characterization (J. Wiley Sons, Inc., 2006)

[35] D.L. Losee. Appl. Phys. Lett., 21, 54 (1972).

[36] D.L. Losee. J. Appl. Phys., 46, 2204 (1975).

[37] J.L. Pautrat, B. Katircioglu, N. Magnea, D. Bensahel, J.C. Pfister, L. Revoil. Solid-St. Electron., 23, 1159 (1980).

[38] A.M. Cowley, H.O. Sorensen. IEEE Trans. Microwave Theory Techn., MTT-14, 588 (1966).

[39] S.M. Sze, K.K. Ng. Physics of Semiconductor Devices (J. Wiley Sons, Inc., 2007) 
[40] G.M. Martin, A. Mitonneau, A. Mircea. Electron. Lett., 13, 191 (1977)

[41] G. Vincent, D. Bois, P. Pinard. J. Appl. Phys., 46, 5173 (1975).

[42] C. Ghezzi. Appl. Phys. A., 26, 191 (1981).

[43] S.R. Forrest, O.K. Kim. J. Appl. Phys., 53, 5738 (1982).

[44] А.В. Мурель, В.Б. Шмагин, В.Л. Крюков, С.С. Стрельченко, Е.А. Суровегина, В.И. Шашкин. ФТП, 51, 1538 (2017). DOI: 10.21883/FTP.2017.11.45107.21 [A.V. Murel, V.B. Shmagin, V.L. Krukov, S.S. Strelchenko, E.A. Surovegina, V.I. Shashkin. Semicond., 51 (11), 1485 (2017). DOI: 10.1134/S1063782617110197]

[45] M. Golosovsky, E. Maniv, D. Davidov, A. Frenkel. IEEE Trans. Instr. Meas., 51, 1090 (2002). DOI: $10.1109 /$ TIM.2002.806006

[46] A. Karbassi, D. Ruf, A.D. Bettermann, C.A. Paulson, D.W. Van der Weide, H. Tanbakuchi, R. Stancliff. Rev. Sci. Instrum., 79, 094706 (2008). DOI: 10.1063/1.2953095

[47] C. Balusek, B. Friedman, B. Oetiker, A. Babajanyan, K. Lee. J. Appl. Phys., 112, 084318 (2012). DOI: 10.1063/1.4759253

[48] D.E. Steinhauer, C.P. Vlahacos, F.C. Wellstood, S.M. Anlage, C. Canedy, R. Ramesh, A. Stanishevsky, J. Melngailis. Rev. Sci. Instrum., 71, 2751 (2000). DOI: 10.1063/1.1150687

[49] J.H. Lee, S. Hyun, K. Char. Rev. Sci. Instrum., 72, 1425 (2001). DOI: $10.1063 / 1.1342032$

[50] K. Lai, W. Kundhikanjana, M. Kelly, Z.X. Shen. Rev. Sci. Instrum., 79, 063703 (2008). DOI: 10.1063/1.2949109

[51] Z. Wei, Y.-T. Cui, E.Y. Ma, S. Johnston, Y. Yang, R. Chen, M. Kelly, Z.-X. Shen, X. Chen. IEEE Trans. Microwave Theory Tech., 64, 1402 (2016).

DOI: 10.1109/TMTT.2016.2537801 